\title{
Comparison of transarterial bland and chemoembolization for neuroendocrine tumours: a systematic review and meta-analysis
}

E. Tai MD PhD, ${ }^{*}$ S. Kennedy MD, ${ }^{*}$ A. Farrell MLIS, ${ }^{\dagger}$ A. Jaberi $M D,{ }^{*}$ J. Kachura MD, ${ }^{*}$ and R. Beecroft MD*

\begin{abstract}
Background Treatment of hepatic metastases from neuroendocrine tumours improves survival and symptom relief. Hepatic arterial embolotherapy techniques include transarterial chemoembolization (TACE) and bland embolization (TAE). The relative efficacy of the techniques is controversial. The purpose of the present study was to use a meta-analysis and systematic review to compare TACE with TAE in the treatment of hepatic metastases.
\end{abstract}

Methods A literature search identified studies comparing TACE and TAE for treatment of hepatic metastases. Outcomes of interest included overall survival (OS), progression-free survival (PFS), radiographic response, complications, and symptom control. The hazard ratios (HRs) and odds ratios (ORs) were estimated and pooled.

Results Eight studies and 504 patients were included. No statistically significant differences between TACE and TAE were observed for os at 1,2, and 5 years or for HRs [1-year OR: 0.72; 95\% confidence interval (CI): 0.27 to 1.94; $p<0.52$; 2-year OR: 0.69 ; $95 \%$ CI: 0.43 to $1.11 ; p<0.12$; 5 -year OR: $0.91 ; 95 \%$ CI: 0.37 to $2.24 ; p<0.85$; HR: $0.96 ; 95 \%$ CI: 0.73 to $1.24 ; p<$ $0.74]$. No statistically significant differences between TACE and TAE were observed for PFS at 1,2 , and 5 years or for HRs (1-year OR: $0.71 ; 95 \%$ CI: 0.38 to $1.55 ; p<0.30 ; 2$-year OR: 0.83 ; $95 \%$ CI: 0.33 to $2.06 ; p<0.69 ; 5$-year OR: $0.91 ; 95 \%$ CI: 0.37 to 2.24; $p<0.85$; HR: $0.99-1.74 ; 95 \%$ CI: 0.74 to $1.73 ; p<0.97$ ). Both techniques are safe and effective for symptom control.

Conclusions No statistically significant differences between TACE and TAE were observed for OS and PFS.

Key Words Neuroendocrine tumours, hepatic metastases, chemoembolization, bland embolization

Curr Oncol. 2020 December:27(6)e537-e546

www.current-oncology.com

\section{INTRODUCTION}

Neuroendocrine tumours (NETs) are the 2nd most common gastrointestinal malignancy after colon cancer. Up to $90 \%$ of patients with NETs present with or develop liver metastases. Patients with hepatic metastases often develop significant morbidity secondary to hormone release and impaired metabolic capacity of the liver ${ }^{1}$. Hepatic metastases are a major determinant of symptoms and survival. Patients with unresectable hepatic metastases treated with supportive care have a 5 -year survival of only $0 \%-22 \%^{2}$.

In patients with liver metastases from a NET, improved survival has been demonstrated with octreotide to regulate symptoms related to hormone secretion and with hepatic arterial embolotherapy techniques. Embolotherapy includes transarterial chemoembolization (TACE) and bland hepatic arterial embolization (TAE). Bland embolization is performed using microscopic embolic particles instilled through the hepatic artery to cut off the tumour's blood supply. Chemoembolization instills an oily emulsion of chemotherapy drugs such as doxorubicin ${ }^{3-11}$.

Current guidelines from the U.S. National Comprehensive Cancer Network, the North American Neuroendocrine Tumor Society, the European Neuroendocrine Tumor Society, and a Canadian consensus report support embolotherapy for symptomatic or progressive hepatic 
metastases with level 2B-3 evidence ${ }^{12-17}$. Of the available embolization approaches, no approach is specifically recommended. It is commonly accepted that TAE and TACE are both associated with favourable results with respect to overall survival (OS) and progression-free survival (PFS).

Most of the literature concerning management of hepatic metastases from NETs consists of retrospective studies with inconsistent outcomes for survival, symptom control, progression, and safety ${ }^{5-8,18,19}$, and few prospective studies have looked at the management of hepatic metastases from NETs ${ }^{20-22}$. In the present systematic review and meta-analysis, we compare TACE with TAE for the treatment of hepatic NET metastases with respect to OS, PFS, radiologic response, symptom control, and complications.

\section{METHODS}

This systematic review and meta-analysis was conducted in accordance with a protocol developed $a$ priori and with the PRISMA (Preferred Reporting Items for Systematic Reviews and Meta-Analyses) statement ${ }^{23}$. Research ethics board approval was not required.

\section{Eligibility Criteria}

Study inclusion criteria for the systematic review were:

Human study

Comparative study with at least 2 arms comparing outcomes between TAE and TACE (either Lipiodol or drug-eluting beads)

Hepatic NET-directed treatment

Exclusion criteria were:

Case report or series reporting fewer than 5 patients Single-arm study

Conference or meeting abstract

Treatment directed at non-neuroendocrine hepatic tumours

\section{Systematic Search and Data Abstraction}

Primary systematic searches for all studies assessing the use of TAE and TACE for the treatment of hepatic NET burden were performed in the MEDLINE (1946 to 5 June 2020), Cochrane (2005 to 5 June 2020), and EMBASE (1974 to 5 June 2020) databases. Supplemental Appendix 1 details the search strategy. Secondary searches were conducted by 2 reviewers (ET, SK), who assessed bibliographies from primary studies, conference and meeting abstracts for subsequent publications, and review papers to identify any additional papers for inclusion. Two reviewers (ET, SK) independently screened abstracts found in both the primary and secondary literature searches. After the initial abstract screen, the full texts of the studies that passed the screening were independently reviewed. In the event of inter-reviewer disagreement, the remaining study authors reached consensus. The reviewers extracted data into a standardized spreadsheet.

\section{Statistical Analyses}

Study outcomes of interest included os, PFS, radiologic response, achievement of symptom control or relief, and treatment-related complications. A meta-analysis was performed when at least two studies assessed at least 1 of the foregoing outcomes. If provided, hazard ratio (HRs) were used for OS and PFS. Otherwise, the HR was estimated using the methods published by Tierney et al. ${ }^{24}$. Meta-analyses were performed using the RevMan software application (version 5.3.5: The Cochrane Collaboration, Copenhagen, Denmark). Results are expressed as odds ratios (ORs) using the number of events per number of patients receiving TACE and TAE. Mantel-Haenszel random-effects models were applied. Heterogeneity was evaluated using the $I^{2}$, which measures the percentage of total variation attributable to heterogeneity rather than to chance. Higgins et al. ${ }^{25}$ suggest values of $25 \%, 50 \%$, and $75 \%$ for low, moderate, and high heterogeneity.

\section{Study Quality Assessment}

Individual study bias was assessed using the Cochrane Risk of Bias tool for randomized controlled trials and the Newcastle-Ottawa scale for non-randomized controlled trials. The Cochrane Risk of Bias tool assesses the appropriate use of random sequence generation, allocation concealment, blinding of participants and personnel, blinding of outcome assessors, incomplete reporting of outcomes data, selective reporting, and other factors ${ }^{26}$. The Newcastle-Ottawa scale is a 9-point rating scale: 4 points for study selection, 2 points for study comparability, and 3 points for study outcomes assessment ${ }^{27}$. A score greater than 5 is indicative of high quality. The reviewers made independent assessments. Disagreements were resolved with the remaining study authors.

\section{RESULTS}

\section{Literature Search and Study Selection}

Figure 1 shows the results of the literature review strategy. The search strategy identified 3001 abstracts. After screening, 2355 publications were excluded, and 28 underwent full-text review. After the full-text review, eight articles met the inclusion criteria.

\section{Study Characteristics}

Table I presents the patient, intervention, and outcome characteristics of the included studies. The systematic review included 593 patients, and the meta-analysis included 470 patients. Of the included patients, 204 underwent chemoembolization with Lipiodol, and 208 underwent TAE for management.

\section{Methodologic Quality of Included Studies}

Table II presents assessments of study quality for the observational studies and randomized controlled trials. Of seven retrospective cohort studies, only the study by Chen et al. ${ }^{29}$ receiving a perfect score $(9 / 9)$ because of propensity score weighting for Eastern Cooperative Oncology Group performance status, tumour grade, tumour primary site, hepatic tumour burden, presence of extrahepatic metastases, indication for embolotherapy, and systemic treatment during follow-up. The remaining six studies received a score of 7 , all having lost 2 points for comparability, 


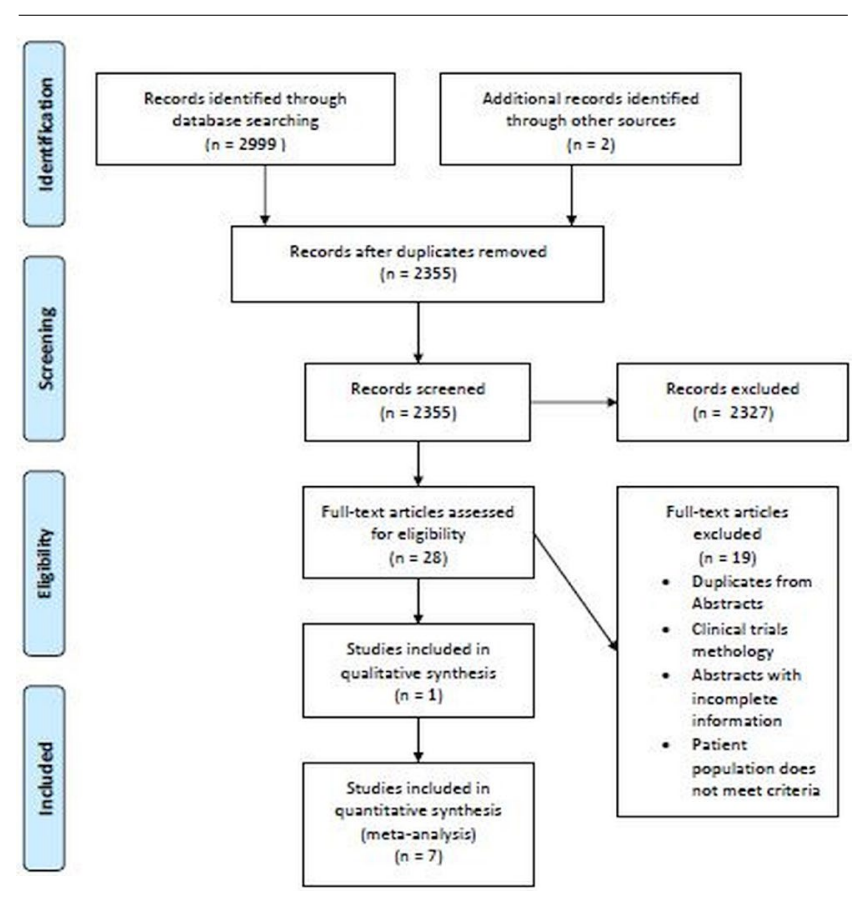

FIGURE 1 PRISMA flow diagram for the included studies.

because no attempt was made to adjust for confounding factors ${ }^{5,6,8,9,19,28}$. The randomized controlled trial by Maire et al. ${ }^{18}$ had a low risk of bias in all categories, and was rated to have an overall low risk of bias.

\section{Overall Survival}

Five studies met the inclusion criteria for an analysis of 1and 2-year os. Two studies were included in the analysis of 5 -year os. Four studies met the inclusion criteria for HR. Four studies were retrospective cohort studies, and one study was a randomized controlled trial $1^{8,9,18,19,29}$. The studies included in the 5-year analysis were both retrospective cohort studies. Overall survival was calculated from time of embolization to time of death and interpolated from Kaplan-Meier curves. Maire et al. ${ }^{18}$ calculated os from time of randomization to time of death, and we assumed that the time from randomization to embolization was negligible. Pericleous et al. ${ }^{8}$ did not specify the criteria for calculating os, and we assumed that it was calculated from the date of embolization.

We observed no statistically significant differences between TACE and TAE at 1 year, 2 years, and 5 years, or in the HR (Figure 2). Although we observed no statistically significant differences between TACE and TAE at 1 year, 2 years, and 5 years, os at 2 years tended to favour TAE [odds ratio (OR): $0.69 ; 95 \%$ confidence interval (CI): 0.43 to $1.11 ; p<0.12$ ].

Groups also reported the median os for TACE and TAE. A meta-analysis could not be performed because the interquartile range was not reported. Median os was generally reported from the time of embolization (supplemental Table 1). Gupta et al. ${ }^{6}$ reported separate values for small-bowel and pancreatic NETs after TACE and TAE. Pitt $e t$ $a l .{ }^{19}$ reported median os from the time of diagnosis, which was 39.1 months for the TAE group and 50.1 months for the
TACE group. A meta-analysis could not be performed because the interquartile range was not reported.

\section{Progression-Free Survival}

Four studies met the inclusion criteria for the meta-analysis of 1- and 2-year PFs $5,18,28,29$. Two studies met the inclusion criteria for the meta-analysis of 5 -year $\mathrm{PFS}^{5,28}$. Five studies met the inclusion criteria for the HR. Typically, PFS was measured using a Kaplan-Meier analysis starting at the time of embolization. Maire et al. ${ }^{18}$ reported PFs from the time of randomization, which was assumed to be negligibly different from the time of embolization. Fiore et al. ${ }^{5}$ did not specify how PFS was calculated; their PFS was assumed to start from the time of embolization. We observed no statistically significant differences in PFS between the TACE group and the TAE group at 1,2, and 5 years or in the HR (Figure 3 ).

Groups also reported the median PFs. A meta-analysis of those results could not be performed because the interquartile range was not reported. The PFS was typically reported from the time of embolization (supplemental Table 2). Maire et al. ${ }^{18}$ reported PFs from the time of randomization. Fiore et al. ${ }^{5}$ and Pericleous et al. ${ }^{8}$ did not report the criteria for measuring the median PFs. Gupta et al. ${ }^{6}$ reported PFS separately for small-bowel and pancreatic NETs. Ruutiainen et al..$^{9}$ reported time to progression, defined as the time from initial stabilization to the first examination showing radiographic progression, and time to treatment failure, defined as the time from initial stabilization to the first progression after the last successful therapy. The median time to progression was 6 months for TAE compared with 12 months for TACE. Median time to treatment failure was 8 months for TAE compared with 33 months for TACE.

\section{Complications}

All studies reported complications. Three studies met the inclusion criteria for major complications ${ }^{9,18,29}$. Complications were typically reported using the Common Terminology Criteria for Adverse Events. A grade of 3 or greater based on the Common Terminology Criteria for Adverse Events was considered a major complication. We observed no statistically significant differences between TACE and TAE for major complications (OR: 1.12; 95\% CI: 0.47 to 2.64 ; $p<0.71$; Figure 4 ).

A meta-analysis of the remaining data could not be performed given that not all studies separated TACE and TAE, and given heterogeneity in the reporting of complications. All papers reported post-embolization syndrome (abdominal pain, fever, nausea, vomiting) as the most common adverse outcome. Other complications included weight loss, infection (sepsis, hepatic abscess), myelosuppression, cardiac complications, and liver enzyme abnormalities ${ }^{5,6,8,9,18,19,28,29}$.

Chen et al. ${ }^{29}$ noted an $86 \%$ complication rate for TAE compared with $80 \%$ for TACE. Ruutiainen et al. ${ }^{9}$ reported an overall $1.4 \% 30$-day mortality rate (3 patients-2 receiving TAE, 1 receiving TACE). Engelman et al. ${ }^{28}$ reported no post-procedure deaths and no differences between TAE and TACE. Fiore et al. ${ }^{5}$ reported no post-procedure deaths, but they observed post-embolization syndrome in $41 \%$ of patients receiving TAE compared with $61 \%$ of patients 


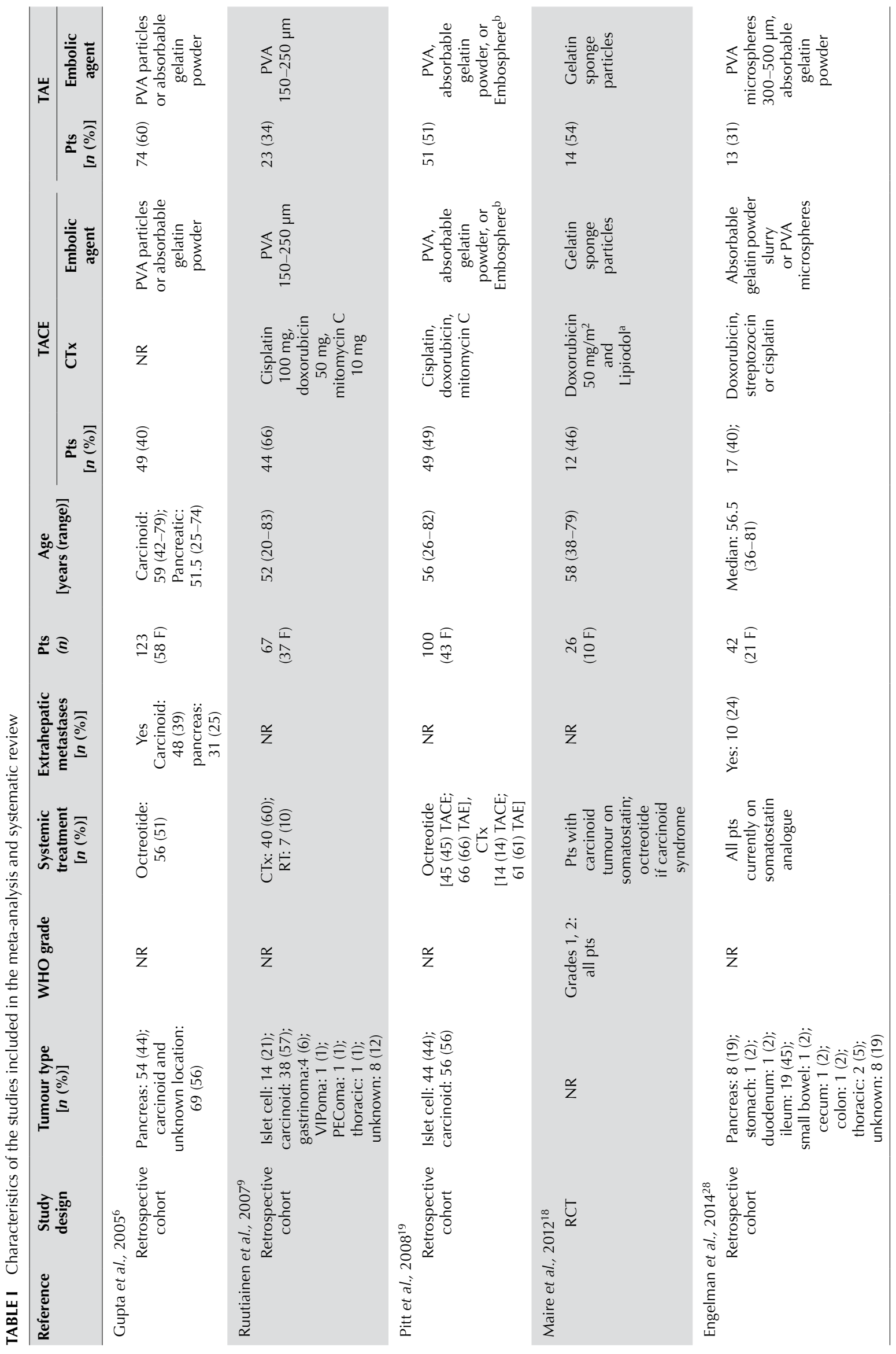




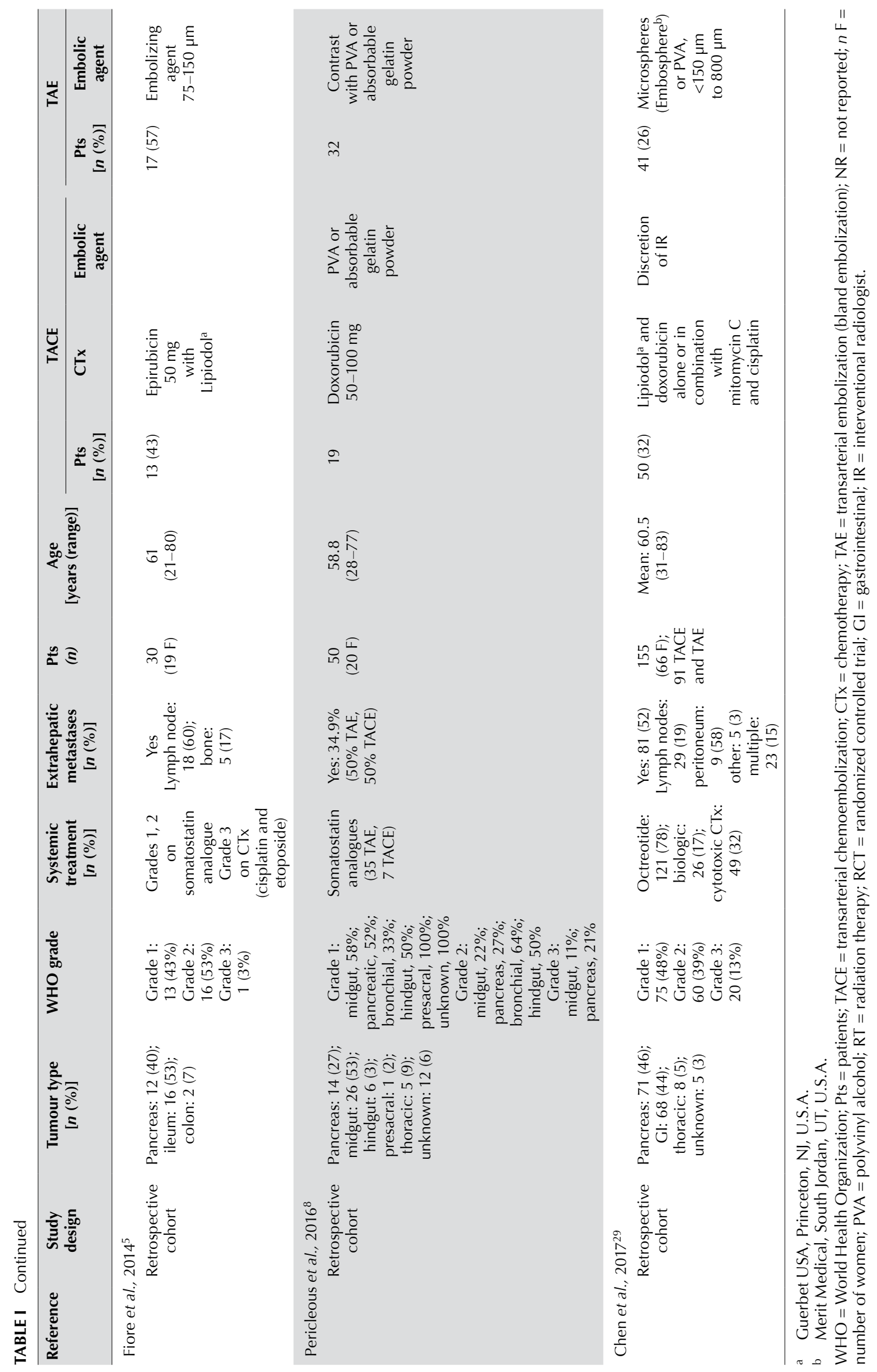




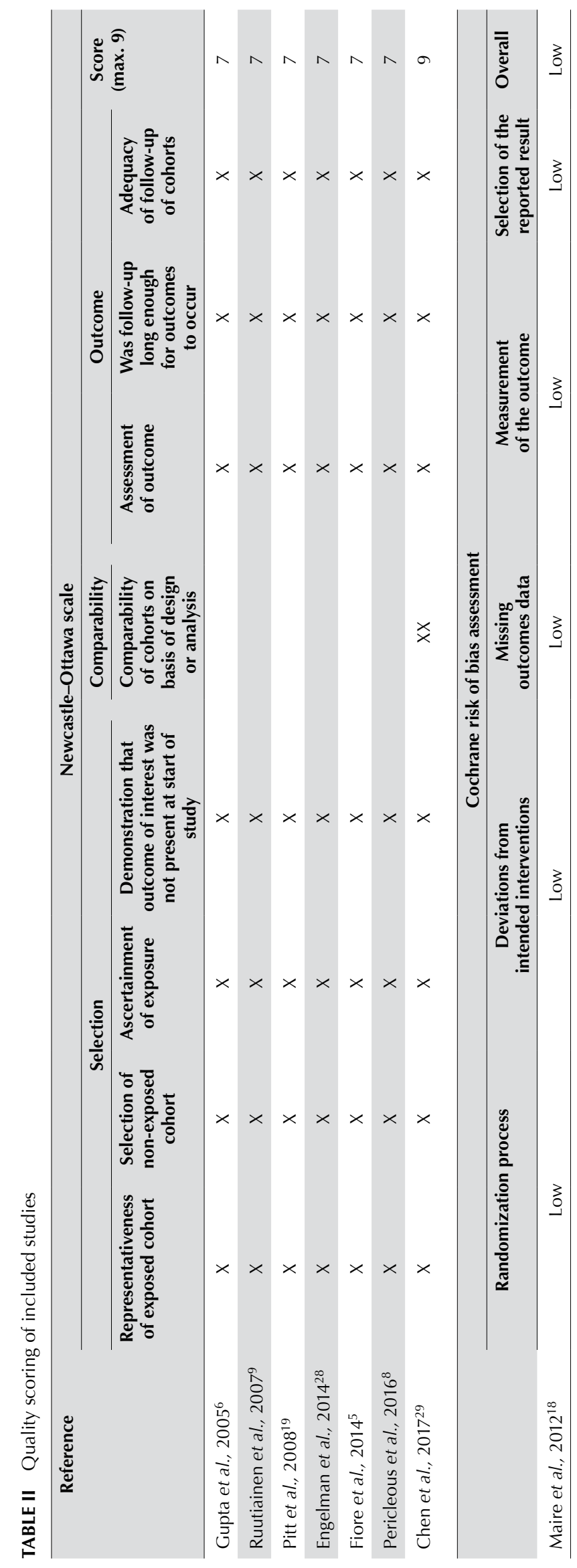

receiving TACE. Gupta et al. ${ }^{6}$ noted an overall serious adverse event rate of $8.5 \%$. The incidence of complications from TACE and TAE were $20 \%$ and $12 \%$ respectively. Maire et al. ${ }^{18}$ noted an overall adverse event rate of $92 \%$ for TACE compared with $86 \%$ for TAE. Pericleous et al. reported a complication rate of $52.5 \%$ and a $16 \%$ serious complication rate that included death. The post-embolization mortality rate was $4 \%{ }^{8}$. Pitt et al. ${ }^{19}$ noted morbidity rates of $2.4 \%$ and $6.6 \%$ for TACE and TAE respectively. The 30 -day mortality rate was $0.8 \%$ for TACE compared with $1.8 \%$ for TAE. Differences between the types of embolization were nonsignificant in all studies.

\section{Radiologic Response}

Radiologic response to treatment was assessed using the Response Evaluation Criteria in Solid Tumours (RECIST), RECIST 1.1, and the World Health Organization (WHO) criteria $^{5,6,8,9,18}$. The RECIST has 4 categories: complete response (disappearance of all target lesions), partial response (at least a $30 \%$ decrease in the sum of the longest diameters of target lesions), stable disease (neither sufficient decrease to qualify for partial response, nor sufficient increase to qualify for progressive disease), and progressive disease (at least a $20 \%$ increase in the sum of the longest diameters of target lesions) ${ }^{30}$. The wHO criteria have 5 categories: complete response (complete disappearance of all recognizable tumour in the liver), partial response (reduction of $\geq 50 \%$ in tumour size), minor response (reduction of $<50 \%$, but $\geq 25 \%$ in tumour size), stable disease (reduction or progression of disease $<25 \%$ ), and progressive disease (increase of $\geq 25 \%$ in tumour size compared with size measured at the time of maximal tumour shrinkage, or appearance of new lesions) ${ }^{31}$. A meta-analysis was not performed given heterogeneity in the reporting of radiologic response.

Pericleous et $a l .{ }^{8}$ used the RECIST 1.1 criteria and reported a response rate of $82 \%$ for TAE compared with $62 \%$ for TACE. Ruutiainen et al. and Fiore $e t$ al. both used RECIST criteria. At 1 month, Ruutiainen et al..$^{9}$ reported $13 \%$ progression, $50 \%$ regression, and $38 \%$ stable disease for $\mathrm{TAE}$ compared with $12 \%, 66 \%$, and $22 \%$ for TACE. Fiore et al. ${ }^{5}$ reported tumour measurements-baseline, $3.3 \pm 1.5 \mathrm{~cm}$; after treatment, $2.2 \pm 1.4 \mathrm{~cm}$-that resulted in a $58.8 \%$ $\pm 28.8 \%$ decrease for TAE. For TACE, pre-treatment measurement was $3.4 \pm 1.7 \mathrm{~cm}$ and post-treatment was $2.2 \pm 1.5 \mathrm{~cm}$, for a $52.7 \% \pm 32 \%$ decrease. The authors observed no statistically significant difference in terms of mean percentage decrease per lesion between TAE and TACE. However, the degree of devascularization of each lesion was higher for TAE than for TACE.

Gupta et al. and Maire et al. both used the WHO criteria to assess radiologic response. Maire et al. ${ }^{18}$ defined radiologic response as the sum of partial response, minor response, and stable disease. No patient achieved a complete response. The response rate for TAE was $92 \%$; it was $100 \%$ for TACE. Gupta et al. ${ }^{6}$ defined responders as experiencing a complete or partial response and separated small-bowel NETs and islet-cell pancreatic tumours. For patients treated with TAE, 34 of 42 patients (81\%) with small-bowel NETs and 8 of 32 patients (25\%) with islet-cell tumour were responders; 12 of 27 (44\%) with small-bowel NETs and 11 of $22(50 \%)$ with islet-cell tumours treated with TACE were responders. 

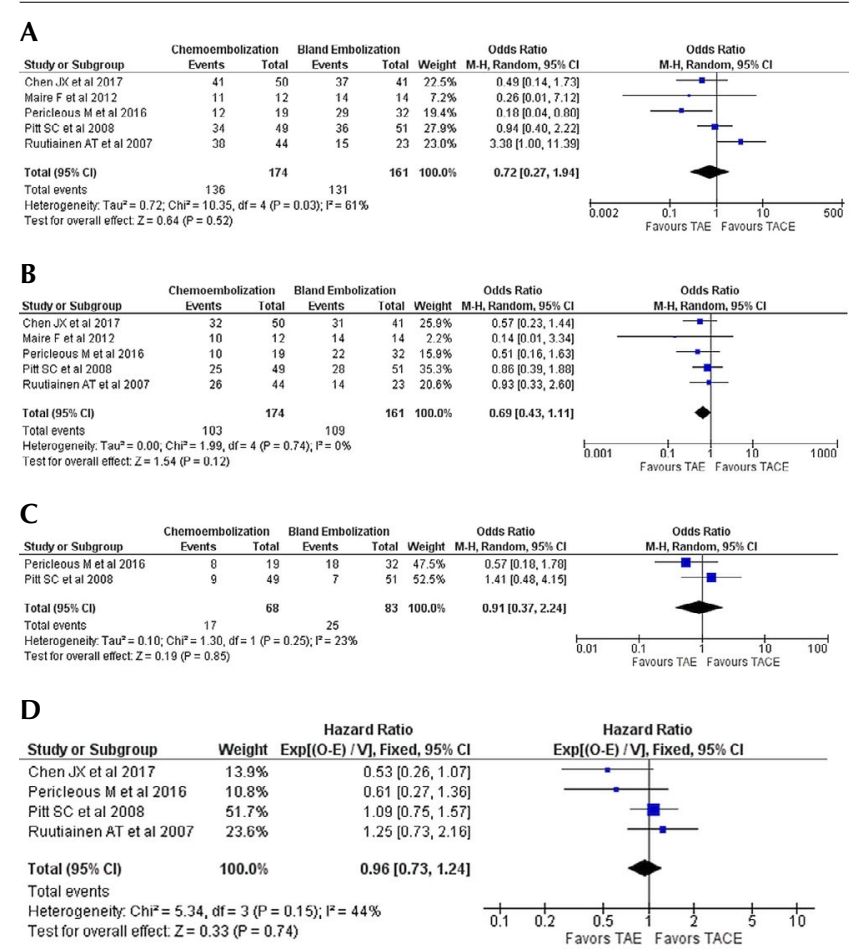

FIGURE 2 Meta-analysis forest plots for (A) 1-year overall survival (OS), (B) 2-year OS, (C) 5-year OS, and (D) hazard ratios for OS. M-H = Mantel-Haenszel; $\mathrm{Cl}=$ confidence interval; TAE = transarterial embolization (bland embolization); TACE $=$ transarterial chemoembolization .

Engelman $e t a l{ }^{28}$ reported radiographic progression based on radiologist interpretation. For patients treated with TAE, 4 of 12 (33\%) experienced a response, and 1 of 12 (8\%) experienced a mixed response; for patients treated with TACE, 7 of 17 (41\%) were responders, 4 of 17 (24\%) experienced a mixed response, and 3 of $17(18 \%)$ progressed after treatment.

\section{Symptom Control}

Four publications reported on symptom control. A metaanalysis could not be performed given heterogeneity in the reporting of outcome. The Fiore et al. ${ }^{5}$ cohort included 21 symptomatic patients, and all experienced symptomatic improvement. Of patients treated with TAE, $7 \mathrm{had}$ carcinoid syndrome, 1 had hypoglycemia, and 4 had Zollinger-Ellison syndrome. Of patients treated with TACE, 4 had carcinoid syndrome, 1 had hypoglycemia, and 2 had Zollinger-Ellison syndrome.

Engelman et al. ${ }^{28}$ assessed symptomatic response by chart review at 3 months for the 27 symptomatic patients who were treated. After the first treatment, 4 of 8 patients receiving TAE (50\%) and 6 of 13 receiving TACE (46\%) experienced symptomatic relief; the difference was not statistically significant.

Pericleous et al. ${ }^{8}$ used a symptom scoring system to evaluate response. Symptomatic response was defined as a reduction of the symptom score by more than $50 \%$. Based on that definition, $75 \%$ of patients receiving TAE and $57 \%$ of those receiving TACE experienced symptomatic relief. The difference was not statistically significant.
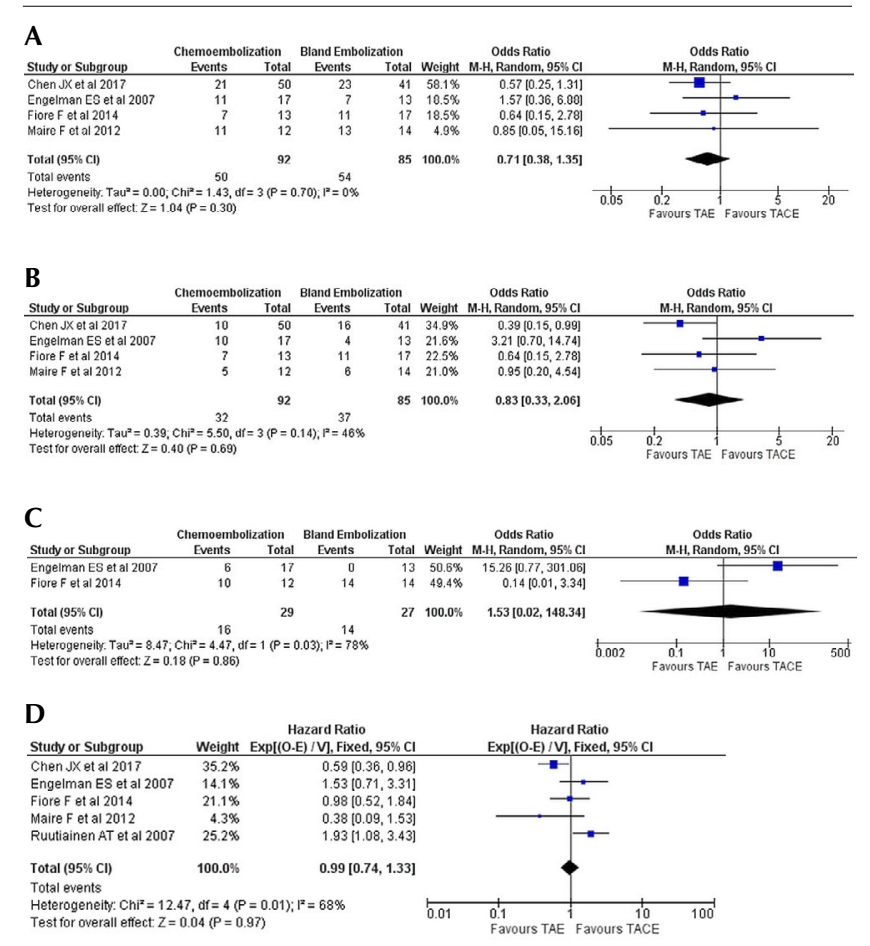

FIGURE 3 Meta-analysis forest plots for (A) 1-year progression-free survival (PFS), (B) 2-year PFS, (C) 5-year PFS, (D) hazard ratios for PFS. $\mathrm{M}-\mathrm{H}=$ Mantel-Haenszel; $\mathrm{Cl}=$ confidence interval; TAE = transarterial embolization (bland embolization); TACE = transarterial chemoembolization .

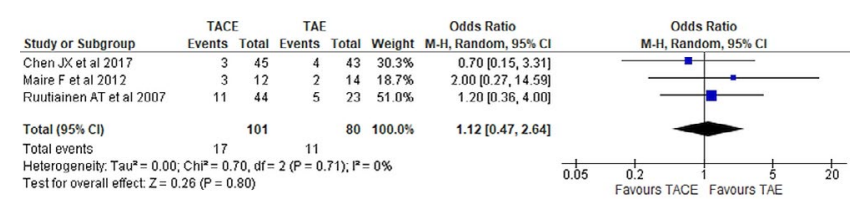

FIGURE 4 Meta-analysis Forest plot for major complications. TAE = transarterial embolization (bland embolization); TACE $=$ transarterial chemoembolization; $\mathrm{M}-\mathrm{H}=$ Mantel-Haenszel; $\mathrm{Cl}$ = confidence interval.

Pitt et al. ${ }^{19}$ noted that $76 \%$ of patients receiving TACE and $69 \%$ of those receiving TAE were symptomatic, and improvement was noted in $86 \%$ and $83 \%$ of those patients respectively. The difference was not statistically significant.

\section{DISCUSSION}

The rarity of NETs limits the number of studies and the quality of the evidence, and presents a challenge to the development of specific treatment algorithms. In addition, several factors-including patient age, male sex, Eastern Cooperative Oncology Group performance status, tumour grade, volume of hepatic burden, and gut compared with pancreatic tumours - have been linked to os ${ }^{4,8-10,18,19,32-36}$.

Several studies have compared embolotherapy techniques for NETs, but they have usually been small retrospective studies because of the prevalence of NETs, and their results about the relative efficacy of TACE compared with TAE have been inconsistent ${ }^{5,6,8,9,19,28,29}$. The Maire et al. ${ }^{18}$ prospective randomized controlled trial compared hepatic arterial chemoembolization with bland embolization in 
metastases from NETs and did not demonstrate a significant difference in outcomes. The study was intended to be a multicentre phase II clinical trial involving 68 patients; however, it recruited only 26 patients despite extending the recruitment period by 2 years. Factors contributing to the difficulty in recruiting included the rarity of the disease, given that NETs have a prevalence of 6.98 per 100,000 population, and the size of the trial, which had only 2 study sites ${ }^{37}$.

The present meta-analysis and systematic review aimed to synthesize and summarize the available data comparing TACE with TAE for hepatic NET metastases. A meta-analysis was performed for the HRs of os and PFS and for the oRs for 1-year, 2-year, and 5-year OS and PFS extracted from Kaplan-Meier curves. Although the results were not statistically significant, preliminary trends suggest that TAE might be more effective than TACE.

In addition, values for os were variable (the median ranged between 21.3 months and 65 months for TAE compared with 25.5 months and 68.7 months for TACE) and equivocal. The median PFS was variable, with values ranging from 12.1-60 months for TAE compared with 8.1-36 months for TACE. A meta-analysis of those results could not be performed because of incomplete data.

Overall, it is clear that, compared with supportive care, TACE and TAE are both effective means of extending PFS and os, eliciting a radiologic response based on objective criteria (WHO, RECIST), and achieving symptom control. However, the relative efficacy of TAE and TACE remain indeterminate after the present meta-analysis and systematic review. Our meta-analysis and review of the literature confirms that both techniques are safe and that, in multiple studies, any difference in the number and severity of complications between TAE and TACE is not statistically significant.

Our results corroborate the systematic review performed by Kanabar et al. ${ }^{38}$ that bland and chemoembolization are safe and effective for treatment of hepatic metastases from NETs. Interestingly, although relative efficacy was not determined, Kanabar noted that TAE trends toward great efficacy in symptom control (TAE, 60\%; TACE, 47.2\%) and PFS (TAE, 22.1 months; TACE, 19.2 months). Given that the literature is equivocal for the use of TAE compared with TACE, increased use of TAE in the treatment of hepatic metastases from NETs should be considered.

All studies included in the present review used conventional TACE with Lipiodol; the use of drug-eluting beads in the treatment of hepatic metastases from NETs has not been well-studied, and our literature search did not identify any studies comparing drug-eluting beads with bland embolization. For treatment of hepatocellular carcinoma, drug-eluting beads, relative to conventional chemoembolization, demonstrated similar effects on PFS and os, while decreasing toxicity ${ }^{39-41}$. One study compared drug-eluting beads with conventional TACE and found a higher symptomatic response with conventional $\mathrm{TACE}^{42}$. As in hepatocellular carcinoma, the toxicity profile of drug-eluting beads is better than the profile of conventional TACE. Additional studies comparing conventional TACE with drug-eluting beads would clarify the comparison.

Radioembolization with ${ }^{90} \mathrm{Y}$ is an alternative hepatic arterial embolotherapy for metastases from NETs. Multiple studies have demonstrated the safety and efficacy of radioembolization ${ }^{21,22,28,43,44}$. Yang et al. ${ }^{43}$ published a systematic review of radioembolization, chemoembolization, and bland embolization, stating that all are effective and safe for the treatment of hepatic metastases from NETs, but comparative data are lacking. Engelman et al. ${ }^{28}$ compared radioembolization with TACE and TAE. That small study enrolled 42 patients and showed no statistically significant difference between its groups for survival, radiographic response, and symptom control. A small study of 43 patients compared radioembolization with drug-eluting beads $^{21}$ and noted similar response rates between the treatments. However, radioembolization was significantly more expensive than embolization using drug-eluting beads. Again, the relative efficacy of radioembolization compared with TACE and TAE has not been determined. The significant added cost associated with radioembolization should also be considered if noninferiority of TACE and TAE can be demonstrated.

Patients with low- to intermediate-grade NETs could also consider peptide receptor radionuclide therapy with ${ }^{177} \mathrm{Lu}$ Dotatate. However, systemic therapy can result in renal toxicity and myelosuppression ${ }^{45,46}$. The LUTIA trial is investigating intra-arterial administration of ${ }^{177} \mathrm{Lu}$ Dotatate for patients with bulky liver metastases ${ }^{47}$.

Our study has several limitations. Because NETs are rare, there is a paucity of literature to guide management of the disease; few studies of NETs have been published. Most studies comparing TACE and TAE are retrospective studies; the single small prospective study enrolled 26 patients. Our meta-analysis and systematic review are limited by that paucity of data.

Statistical measures of heterogeneity were variable in the included studies ( $I^{2}$ values ranged from $0 \%$ to $61 \%$, low to moderate-high). That heterogeneity could relate to multiple factors, including tumour grading, techniques for TACE and TAE, the type of NET, the presence of extrahepatic metastases, and use of other treatments.

The WHO tumour grading was restructured in 2010, resulting in a more objective and reproducible result compared with results emerging from the pathologic grading in the 2000-2004 WHO criteria. Two of the included studies, Gupta et al. ${ }^{6}$ and Ruutiainen et al. ${ }^{9}$, used the old pathologic grading system, affecting comparison of the baseline characteristics of the patients.

Significant heterogeneity in chemoembolization technique was observed not only between studies, but also within studies. Chemotherapeutic agents used included doxorubicin, mitomycin C, cisplatin, streptozocin, and epirubicin. The use of embolic agents was also variable, with use of polyvinyl alcohol, Gelfoam (Pfizer, New York, NY, U.S.A.), and microspheres, with particle size varying in the range $150-800 \mu \mathrm{m}$ not only within studies, but between studies as well. There was variation in the dose of the chemotherapeutic agent, the combination of drugs, and the timing and number of treatments.

The RETNET phase II clinical trial (NCT02724540 at https://ClinicalTrials.gov/ $)^{20}$ in 180 patients is designed to compare conventional chemoembolization with doxorubicin drug-eluting bead chemoembolization and with bland embolization for the treatment of metastases from NETs. At a preliminary analysis, recruitment into 
the drug-eluting bead arm was halted because of safety concerns ${ }^{48}$. Outcomes of interest include hepatic PFS, symptoms, quality of life, toxicity, and adverse events. The authors are also interested in examining the difference between gut and pancreatic NETs. Both Ruutiainen et al. ${ }^{9}$ and Gupta et al. ${ }^{6}$ suggest that prognosis is better for patients with small-bowel NETs. Preliminary data from Gupta et al. suggest that small-bowel NETs and pancreatic islet-cell NETs might respond differently to TACE and TAE. The RETNET trial is projected to be completed in 2021; its results should aid in guiding management of hepatic metastases from NETs and in addressing many of the deficiencies in the literature and the limitations observed in the present meta-analysis and systematic review. The RETNET results and subgroup analyses might also propose additional avenues of investigation to clarify optimal management of patients with NET and hepatic metastases.

\section{SUMMARY}

We observed no statistically significant difference between TACE and TAE for OS and PFS in the treatment of hepatic metastases from NETs. The meta-analysis suggests a trend favouring TAE. Both techniques are safe and effective.

\section{CONFLICT OF INTEREST DISCLOSURES}

We have read and understood Current Oncology's policy on disclosing conflicts of interest, and we declare that we have none.

\section{AUTHOR AFFILIATIONS}

*Division of Interventional Radiology, Joint Department of Medical Imaging, University Health Network, Toronto, ON; ${ }^{\dagger}$ Library and Information Services, Toronto General Hospital, Toronto, ON.

\section{REFERENCES}

1. Gupta S. Intra-arterial liver-directed therapies for neuroendocrine hepatic metastases. Semin Intervent Radiol 2013; 30:28-38.

2. Chamberlain RS, Canes D, Brown KT, et al. Hepatic neuroendocrine metastases: does intervention alter outcomes? $\mathrm{J} \mathrm{Am}$ Coll Surg 2000;190:432-45.

3. Barbier CE, Garske-Roman U, Sandstrom M, Nyman R, Granberg $\mathrm{D}$. Selective internal radiation therapy in patients with progressive neuroendocrine liver metastases. EurJ Nucl Med Mol Imaging 2016;43:1425-31.

4. Dong XD, Carr BI. Hepatic artery chemoembolization for the treatment of liver metastases from neuroendocrine tumors: a long-term follow-up in 123 patients. Med Oncol 2011;28(suppl 1):S286-90.

5. Fiore F, Del Prete M, Franco R, et al. Transarterial embolization (TAE) is equally effective and slightly safer than transarterial chemoembolization (TACE) to manage liver metastases in neuroendocrine tumors. Endocrine 2014;47:177-82.

6. Gupta S, Johnson MM, Murthy R, et al. Hepatic arterial embolization and chemoembolization for the treatment of patients with metastatic neuroendocrine tumors: variables affecting response rates and survival. Cancer 2005;104:1590-602.

7. Memon K, Lewandowski RJ, Riaz A, Salem R. Chemoembolization and radioembolization for metastatic disease to the liver: available data and future studies. Curr Treat Options Oncol 2012;13:403-15.

8. Pericleous M, Caplin ME, Tsochatzis E, Yu D, Morgan-Rowe L, Toumpanakis C. Hepatic artery embolization in advanced neuroendocrine tumors: efficacy and long-term outcomes. Asia Pac J Clin Oncol 2016;12:61-9.
9. Ruutiainen AT, Soulen MC, Tuite CM, et al. Chemoembolization and bland embolization of neuroendocrine tumor metastases to the liver. J Vasc Interv Radiol 2007;18:847-55.

10. Sofocleous CT, Petre EN, Gonen M, et al. Factors affecting periprocedural morbidity and mortality and long-term patient survival after arterial embolization of hepatic neuroendocrine metastases. J Vasc Interv Radiol 2014;25:22-30.

11. Bhagat N, Reyes DK, Lin M, et al. Phase II study of chemoembolization with drug-eluting beads in patients with hepatic neuroendocrine metastases: high incidence of biliary injury. Cardiovasc Intervent Radiol 2013;36:449-59.

12. Boudreaux JP, Klimstra DS, Hassan MM, et al. The NANETS consensus guideline for the diagnosis and management of neuroendocrine tumors: well-differentiated neuroendocrine tumors of the jejunum, ileum, appendix, and cecum. Pancreas 2010;39:753-66.

13. Kennedy A, Bester L, Salem R, Sharma RA, Parks RW, Ruszniewski P on behalf of the NET-Liver-Metastases Consensus Conference. Role of hepatic intra-arterial therapies in metastatic neuroendocrine tumours (NET): guidelines from the NET-Liver-Metastases Consensus Conference. $H P B$ (Oxford) 2015;17:29-37.

14. Kulke MH, Anthony LB, Bushnell DL, et al. on behalf of the North American Neuroendocrine Tumor Society (NANETS). NANETS treatment guidelines: well-differentiated neuroendocrine tumors of the stomach and pancreas. Pancreas 2010;39:735-52.

15. Kulke MH, Shah MH, Benson AB 3rd, et al. Neuroendocrine tumors, version 1.2015. JNatl Compr Canc Netw2015;13:78-108.

16. Pavel M, O'Toole D, Costa F, et al. on behalf of the Vienna Consensus Conference participants. ENETS consensus guidelines update for the management of distant metastatic disease of intestinal, pancreatic, bronchial neuroendocrine neoplasms (NEN) and NEN of unknown primary site. Neuroendocrinology 2016;103:172-85.

17. Singh S, Asa SL, Dey C, et al. Diagnosis and management of gastrointestinal neuroendocrine tumors: an evidence-based Canadian consensus. Cancer Treat Rev 2016;47:32-45.

18. Maire F, Lombard-Bohas C, O'Toole D, et al. Hepatic arterial embolization versus chemoembolization in the treatment of liver metastases from well-differentiated midgut endocrine tumors: a prospective randomized study. Neuroendocrinology 2012;96:294-300.

19. Pitt SC, Knuth J, Keily JM, et al. Hepatic neuroendocrine metastases: chemo- or bland embolization? J Gastrointest Surg 2008;12:1951-60.

20. Chen JX, Wileyto EP, Soulen MC. Randomized embolization trial for neuroendocrine tumor metastases to the liver (RETNET): study protocol for a randomized controlled trial. Trials 2018;19:390.

21. Whitney R, Valek V, Fages JF, et al. Transarterial chemoembolization and selective internal radiation for the treatment of patients with metastatic neuroendocrine tumors: a comparison of efficacy and cost. Oncologist 2011;16:594-601.

22. Rhee TK, Lewandowski RJ, Liu DM, et al. ${ }^{90}$ Y Radioembolization for metastatic neuroendocrine liver tumors: preliminary results from a multi-institutional experience. Ann Surg 2008;247:1029-35.

23. Liberati A, Altman DG, Tetzlaff $J$, et al. The PRISMA statement for reporting systematic reviews and meta-analyses of studies that evaluate healthcare interventions: explanation and elaboration. BMJ 2009;339:b2700.

24. Tierney JF, Stewart LA, Ghersi D, Burdett S, Sydes MR. Practical methods for incorporating summary time-to-event data into meta-analysis. Trials 2007;8:16.

25. Higgins JP, Thompson SG, Deeks JJ, Altman DG. Measuring inconsistency in meta-analyses. BMJ 2003;327:557-60. 
26. Higgins JP, Altman DG, Gotzsche PC, et al. The Cochrane Collaboration's tool for assessing risk of bias in randomised trials. BMJ 2011;343:d5928.

27. Wells GA, Shea B, O'Connell D, et al. The Newcastle-Ottawa Scale (NOS) for Assessing the Quality of Nonrandomised Studies in Meta-analyses. Ottawa, ON: The Ottawa Hospital Research Institute; 2019. [Available online at: http://www.ohri.ca/ programs/clinical_epidemiology/oxford.asp; cited 23 May 2019]

28. Engelman ES, Leon-Ferre R, Naraev BG, et al. Comparison of transarterial liver-directed therapies for low-grade metastatic neuroendocrine tumors in a single institution. Pancreas 2014;43:219-25.

29. Chen JX, Rose S, White SB, et al. Embolotherapy for neuroendocrine tumor liver metastases: prognostic factors for hepatic progression-free survival and overall survival. Cardiovasc Intervent Radiol 2017;40:69-80.

30. Therasse P, Arbuck SG, Eisenhauer EA, et al. New guidelines to evaluate the response to treatment in solid tumors. European Organization for Research and Treatment of Cancer, National Cancer Institute of the United States, National Cancer Institute of Canada. J Natl Cancer Inst 2000;92:205-16.

31. World Health Organization (WHO). WHO Handbook for Reporting Results of Cancer Treatment. Geneva, Switzerland: WHO; 1979.

32. Yao JC, Hassan M, Phan A, et al. One hundred years after "carcinoid": epidemiology of and prognostic factors for neuroendocrine tumors in 35,825 cases in the United States. J Clin Oncol 2008;26:3063-72.

33. Lepage C, Rachet B, Coleman MP. Survival from malignant digestive endocrine tumors in England and Wales: a population-based study. Gastroenterology 2007;132:899-904.

34. Sommer WH, Ceelen F, Garcia-Albeniz X, et al. Defining predictors for long progression-free survival after radioembolisation of hepatic metastases of neuroendocrine origin. Eur Radiol 2013;23:3094-103.

35. Strosberg JR, Choi J, Cantor AB, Kvols LK. Selective hepatic artery embolization for treatment of patients with metastatic carcinoid and pancreatic endocrine tumors. Cancer Control 2006;13:72-8.

36. Ho AS, Picus J, Darcy MD, et al. Long-term outcome after chemoembolization and embolization of hepatic metastatic lesions from neuroendocrine tumors. AJR Am J Roentgenol 2007;188:1201-7.
37. Dasari A, Shen C, Halperin D, et al. Trends in the incidence, prevalence, and survival outcomes in patients with neuroendocrine tumors in the United States. JAMA Oncol 2017;3:1335-42.

38. Kanabar R, Barriuso J, McNamara MG, et al. Liver embolisation for patients with neuroendocrine neoplasms: systematic review. Neuroendocrinology 2020;:[Online ahead of print].

39. Golfieri R, Giampalma E, Renzulli M, et al. Randomised controlled trial of doxorubicin-eluting beads vs conventional chemoembolisation for hepatocellular carcinoma. BrJCancer 2014;111:255-64.

40. Lammer J, Malagari K, Vogl T, et al. Prospective randomized study of doxorubicin-eluting-bead embolization in the treatment of hepatocellular carcinoma: results of the PRECISION V study. Cardiovasc Intervent Radiol 2010;33:41-52.

41. Vogl TJ, Lammer J, Lencioni R, et al. Liver, gastrointestinal, and cardiac toxicity in intermediate hepatocellular carcinoma treated with PRECISION TACE with drug-eluting beads: results from the PRECISION v randomized trial. AJR Am J Roentgenol 2011;197:W562-70.

42. Makary MS, Kapke J, Yildiz V, Pan X, Dowell JD. Conventional versus drug-eluting bead transarterial chemoembolization for neuroendocrine tumor liver metastases. J Vasc Interv Radiol 2016;27:1298-304.

43. Yang TX, Chua TC, Morris DL. Radioembolization and chemoembolization for unresectable neuroendocrine liver metastases-a systematic review. Surg Oncol 2012;21:299-308.

44. Devcic Z, Rosenberg J, Braat AJ, et al. The efficacy of hepatic ${ }^{90} \mathrm{Y}$ resin radioembolization for metastatic neuroendocrine tumors: a meta-analysis. J Nucl Med 2014;55:1404-10.

45. AlsadikS, YusufS, Al-Nahhas A. Peptide receptor radionuclide therapy for neuroendocrine tumours. Clin Translat Imag 2018;6:101-11.

46. Shah MH, Goldner WS, Halfdanarson TR, et al. NCCN guidelines insights: neuroendocrine and adrenal tumors, version 2.2018. J Natl Compr Cancer Netw 2018;16:693-702.

47. Ebbers SC, Braat AJAT, Moelker A, Stokkel MPM, Lam MGEH, Barentsz MW. Intra-arterial versus standard intravenous administration of lutetium-177-DOTA-octreotate in patients with NET liver metastases: study protocol for a multicenter, randomized controlled trial (LUTIA trial). Trials 2020;21:141.

48. Soulen M, White S, Fidelman N, et al. Randomized Embolization Trial for NeuroEndocrine Tumors (RETNET): first safety report [abstract 105] . J Vasc Intervent Radiol 2019;30:S49-50. 